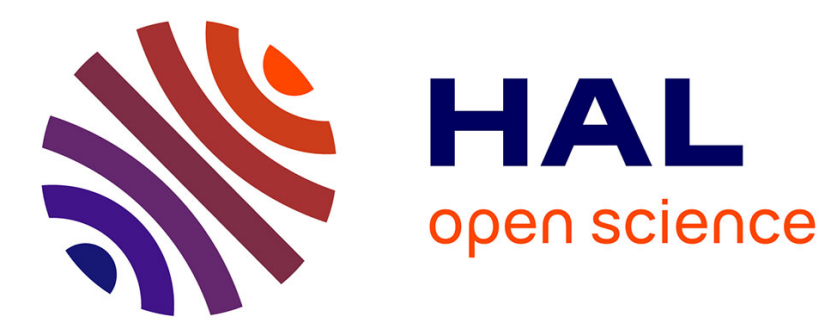

\title{
Stacking fault formation created by plastic deformation at low temperature and small scales in silicon
}

Laurent Pizzagalli, J Godet, S Brochard, H J Gotsis, Tristan Albaret

\section{To cite this version:}

Laurent Pizzagalli, J Godet, S Brochard, H J Gotsis, Tristan Albaret. Stacking fault formation created by plastic deformation at low temperature and small scales in silicon. Physical Review Materials, 2020, 10.1103/PhysRevMaterials.4.093603 . hal-02990149

\section{HAL Id: hal-02990149 \\ https://hal.science/hal-02990149}

Submitted on 5 Nov 2020

HAL is a multi-disciplinary open access archive for the deposit and dissemination of scientific research documents, whether they are published or not. The documents may come from teaching and research institutions in France or abroad, or from public or private research centers.
L'archive ouverte pluridisciplinaire HAL, est destinée au dépôt et à la diffusion de documents scientifiques de niveau recherche, publiés ou non, émanant des établissements d'enseignement et de recherche français ou étrangers, des laboratoires publics ou privés. 


\title{
Stacking fault formation created by plastic deformation at low temperature and small scales in silicon
}

\author{
L. Pizzagalli,* J. Godet, and S. Brochard \\ Departement of Physics and Mechanics of Materials, \\ Institut $P^{\prime}$, CNRS UPR 3346, Université de Poitiers, SP2MI, \\ Boulevard Marie et Pierre Curie, TSA 41123, 86073 Poitiers Cedex 9, France \\ H. J. Gotsis and T. Albaret \\ Univ Lyon, UCB Lyon 1, ILM UMR 5306, 69621 Villeurbanne, France
}

(Dated:)

\begin{abstract}
Several studies have recently reported the formation of stacking faults in silicon compressed at low temperatures and high stresses. This observation contradicts the generally accepted framework for the plastic deformation of silicon. We propose here an original plasticity mechanism that could potentially explain stacking fault formation in these conditions: the nucleation and migration of a partial edge dislocation with Burgers vector $1 / 3\langle 112\rangle$. These results are obtained thanks to a multiscale approach combining three computational methods. Dislocation nucleation is determined by molecular dynamics in both a nanowire and a $2 \mathrm{D}$ slab. The latter results are used as inputs for hybrid MD/DFT "learn on the fly" calculations, allowing for studying the dynamical propagation of the dislocation. Selected configurations at different steps are next used for initiating nudged elastic band density functional theory calculations. These calculations revealed that the dislocation displacement mechanism depends on the compression strain. For low values, a dangling bond is temporarily created in the core, resulting in high activation energies. For compression strains larger than about $8 \%$, the reduction of the interlayer distance allows for a more complex displacement mechanism with no dangling bonds in the dislocation core and a significant decrease of the activation energy.
\end{abstract}

Keywords: Dislocations; Stacking faults; Plastic deformation; Elemental semiconductors; Nanostructures; Multiscale Modeling; Density functional theory; Molecular dynamics

\section{INTRODUCTION}

Thanks to a large amount of dedicated investigations, it seems that the mechanical properties of silicon are now well known. In usual conditions, i.e. for a mechanical stress exerted on a macroscopic sample at room temperature, silicon is brittle as expected for a covalent material. At high temperature, roughly above $600{ }^{\circ} \mathrm{C}$, the plastic deformation proceeds by $30^{\circ}$ and $90^{\circ}$ Shockley partial dislocations located in $\{111\}$ glide set planes, the narrowly spaced planes along the $\langle 111\rangle$ direction [1-3]. Leading and trailing partial dislocations are separated by an intrinsic stacking fault. Plasticity can also be obtained at low temperatures by using a confining pressure which prevents cracks opening and fracture propagation [4]. In these conditions, both experiments and numerical simulations reveal that dislocations are not dissociated and glide in the widely spaced $\{111\}$ 'shuffle' set of planes [58]. The non-dissociation of these dislocations is related to the prohibitive energetic cost of a stacking fault in the shuffle $\{111\}$ planes [9]. There is currently a large consensus in the community regarding the existence of separate shuffle and glide dislocation regimes, although the transition from one to the other is not fully explained [10, 11].

Reducing the characteristic dimensions can also lead to plastic deformation of silicon at low temperatures [12].

* laurent.pizzagalli@univ-poitiers.fr
This has been demonstrated in the case of the uniaxial compression at room temperature of nanopillars [1316] and nanoparticles [14, 17, 18]. An intriguing aspect in some of these studies is the report of $\{111\}$ stacking faults. For instance, they are observed in compressed nanometric silicon nanocubes [17]. The authors proposed that below a given size there could be a transition from shuffle perfect to glide partial dislocations, but it is not clear why such a transition would occur. Twinning was also reported in bent silicon nanowires [19]. More recently, Merabet et al. performed high resolution transmission electron microscopy experiments on compressed nanopillars, and reported the presence of stacking faults and complex dislocation patterns [16]. A comparison with atomistic simulations suggests that the stacking faults could be associated with shuffle partial dislocations [20]. Similar defects were predicted in an earlier study [21], but never observed since. As yet there is therefore no clear consensus nor firmly established conclusions regarding the formation mechanisms of these stacking faults.

There is a crucial need for a better understanding of materials properties at small scales, and the mechanical behavior of silicon is no exception. Silicon-based nano-objects are particularly promising in numerous applications, such as nano-electro-mechanical systems [22] or Li-ion batteries [23-25]. Furthermore, silicon nanostructures are also key systems for elastic strain engineering [26]. In all these examples, relevant conditions of 
use are low temperatures and high stresses. Therefore improving our knowledge on plasticity properties of silicon in this regime is important both for fundamental and applied sciences.

In the present study we describe an original mechanism leading to stacking fault formation during the plastic deformation of silicon at high stress and room temperature. A similar mechanism was observed in an earlier investigation of dislocation nucleation [27] by some of the present authors. This study was based on classical molecular dynamics calculations, and at that time there were doubts about the reliability of the used interatomic potentials in the high stress regime. The aforementioned new experimental evidences of stacking fault formation in silicon at room temperature, as well as a recent work on dislocation nucleation [28], motivated us to reopen the case, but this time using more accurate electronic structure calculation techniques. To minimize errors associated to small system sizes [8], we develop a multiscale approach combining classical molecular dynamics (MD) simulations, the "Learn-On-The-Fly" (LOTF) technique, density functional theory (DFT) calculations combined with the Nudged Elastic Band (NEB) method.

The paper is organized in a standard way. The multiscale methodology and the three calculation frameworks used in this work are described in the section II. All our results are reported then discussed in the sections III and IV. In the last section, we summarize the results.

\section{METHODS}
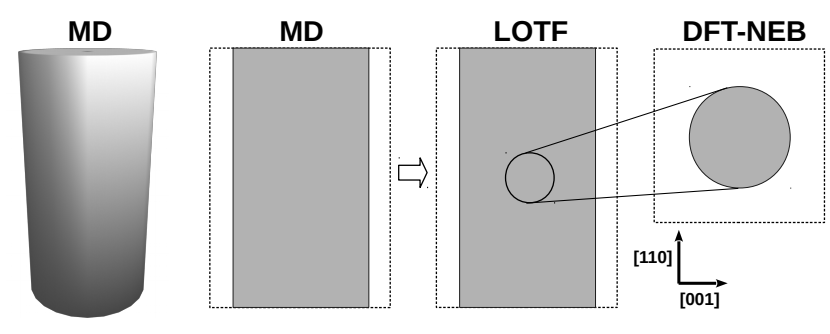

FIG. 1. Multiscale modeling framework. From left to right: $\langle 110\rangle$ oriented cylindrical nanowire (MD calculations), 2Dperiodic slabs (both MD and LOTF calculations), 1D-periodic system (DFT-NEB). The dashed lines represent the supercells used in periodic boundary calculations.

The multiscale methodology employed in this work is sketched in Fig. 1. First, MD calculations are performed for large nanowires and small 2D-periodic slabs. The output of MD slab calculations leading to dislocation nucleation and stacking fault formation is next used as input for more time-consuming LOTF simulations. The latter allow for exploring the dynamics of dislocation migration with accuracy close to first principles. Finally, regions of interest from LOTF results are extracted and employed to feed DFT-NEB calculations, to determine the variation of activation energy as a function of the applied compression strain.

\section{A. MD simulations}

We used the code LAMMPS [29, 30] to perform the classical MD calculations. Silicon is described by the SWm interatomic potential [31], an improved version of the original Stillinger-Weber potential for modeling defects and plastic behavior. The lattice parameter is $a_{0}=5.431 \AA$. A timestep in the range $0.5-1$ fs is used, which allows for reasonable simulation times while ensuring energy conservation.

Two different models were considered. One is a $\langle 110\rangle$ oriented cylindrical nanowire with a length of $23.4 \mathrm{~nm}$ and a diameter of $11.6 \mathrm{~nm}$ including 105209 atoms. An amorphous coating is created by annealing the nanowire at $3000 \mathrm{~K}$ during 5 ps, leading to surface melting, followed by a linear decrease of the temperature to $300 \mathrm{~K}$ for another 5 ps. This is intended to reproduce the disordered shell which is often observed at the surface of silicon nanopillars [16]. Next, the resulting system is compressed according to the following procedure. At one end of the nanowire, six $\langle 110\rangle$ atomic layers are frozen in their initial positions. A flat punch, modeled by a repulsive planar force field [32], is applied on the other end. The flat punch position is modified at each timestep with a strain rate equal to $10^{6} \mathrm{~s}^{-1}$. The temperature is controlled by a Nosé-Hoover thermostat [33, 34].

The second model is a 2D silicon slab, with orientations $\hat{x}=[001], \hat{y}=[1 \overline{1} 0]$, and $\hat{z}=[110]$. The dimensions are $7.5 a_{0} \times a_{0} / \sqrt{2} \times 10 a_{0} \sqrt{2}$. Periodic boundary conditions are enforced only for $\hat{y}$ and $\hat{z}$ orientations, thus yielding a slab with (100) surfaces. A step is created in one of these surfaces to act as a stress localization center and to favor dislocation nucleation [35]. The slab is compressed by decreasing the supercell dimension along $\hat{z}$ at regular times during a temperature-controlled simulation. At each compression step a remapping of atomic positions is performed in order to avoid deformation waves.

\section{B. LOTF}

A hybrid method combining forces computed from both quantum calculations and a classical empirical potential is used to investigate the mechanisms obtained from classical MD calculations. Reaching a better evaluation of the forces is indeed especially important in the active region where the defect propagates with a series of bond breaking and rebonding events, and where the accuracy of a fully classical model is questionable. In this work we use an implementation of the LOTF method that was already described in several previous works and that is especially well suited for silicon based systems [36-39]. 
The principle of the method as well as the algorithm that selects the quantum region are detailed in appendix A. All remaining technical details are identical to those already reported in [39] and in its supplementary material. In the present implementation the initial system is imported from MD calculations using the $2 \mathrm{D}$ slab model, with similar dimensions and orientations. Only the length along $\hat{y}$ was multiplied by two to obtain a cell dimension larger than twice the potential cut-off. The lattice parameter used in LOTF calculations is $a_{0}=5.421$ $\AA$.

\section{DFT-NEB calculations}

The DFT calculations were carried out using the Quantum ESPRESSO suite of codes [40]. The standard Perdew-Burke-Ernzerhof (PBE) functional is used to determine exchange-correlation contributions [41]. The electron-ion interactions are computed thanks to the fullpotential projector augmented wave PAW method [42]. The electronic structure convergence was tested on a silicon bulk system with a dense k-points grid. Plane-wave and charge density cutoffs of 25 Ry and 200 Ry yielded a lattice constant $a_{0}=5.4698 \AA$ and a bulk modulus of 91.2 GPa.

For modeling the defect, an orthorhombic supercell of dimensions $42 \AA \times a_{0} \sqrt{2} \times 42 \AA$, including 177 atoms, is chosen so as to allow periodicity only along the dislocation line (corresponding to the $\hat{y}$ axis of the slab in MD simulations). For the two other directions, the supercell is large enough to ensure a minimum distance of $9 \AA$ between consecutive replica, which is large enough to suppress potential interactions (Fig. 1). The electronic structure calculations are carried out using a $1 \times 4 \times 1$ Monkhorst-Pack grid [43] for the Brillouin zone sampling, and the Methfessel-Paxton smearing scheme [44]. More details for the model can be found in section III C.

The Broyden-Fletcher-Goldfarb-Shanno (BFGS) approach is employed to relax ions for single systems, with a convergence threshold of $2 \times 10^{-3} \mathrm{eV} \AA^{-1}$. For DFTNEB calculations [45], seven replicas are considered, of which five are optimized using the quick-min algorithm, a convergence threshold of $0.05 \mathrm{eV} \AA^{-1}$, and spring constants of 0.04 hartrees. After convergence, a second DFTNEB calculation was run with the climbing image technique [46], in order to accurately determine the transition state.

\section{RESULTS}

In the following sections the results of the different simulations are described and analyzed.

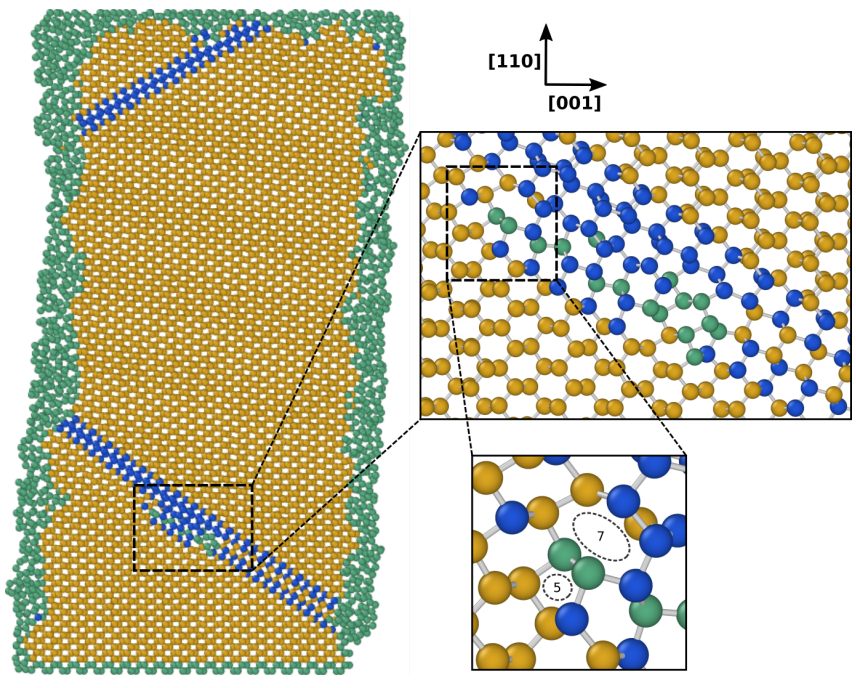

FIG. 2. View of a $5 \AA$ thick slice in the center of the nanowire, for a compression strain equal to $9.873 \%$. The spheres represent silicon atoms, with colors according to their environment determined with the polyedral template matching (PTM) method [47]. Gold: cubic diamond, blue: hexagonal diamond, green: unidentified. Two crystalline regions have been plastically deformed, leaving stacking faults revealed by the presence of hexagonal diamond atoms. The right pictures show enlarged views of the framed areas.

\section{A. Nanowire}

We first focus on the nanowire compressed by a flat punch at $300 \mathrm{~K}$. For low strain values, the deformation of the crystalline core remains elastic. At $9.36 \%$, the plastic deformation of the crystalline core starts by the nucleation of a dislocation loop from the top of the nanowire. The initiation of plastic deformation in the vicinity of the flat punch is reported in several studies $[16,20,48]$. The dislocation slips in the (111) plane and escapes laterally from the nanowire, leaving a stacking fault behind (Fig. 2). At $9.87 \%$ a second slip system is activated with the nucleation from the base of the nanowire of a dislocation loop expanding in a $(11 \overline{1})$ plane. Secondary loops are next nucleated in adjacent planes. As for the first event, dislocation loops expansion leads to stacking faults formation and twinning (Fig. 2). This deformation mode is similar to the one reported in an earlier study [27].

In the traditional picture of dislocations in silicon, stacking faults are exclusively associated to Shockley partial dislocations with $1 / 6\langle 112\rangle$ Burgers vectors and located in glide set of $\{111\}$ planes. Although dislocations identified in the present work also glide in $\{111\}$ planes, we determine the Burgers vector to be equal to $1 / 3\langle 112\rangle$. The leading part of the loop front has an edge character with a line along $\langle 110\rangle$. Enlarged views of this region and of a dislocation core are shown in Fig. 2. The core is characterized by a 7 -atom ring sharing two atoms with a 5-atom ring, with no dangling bonds, and appears to be 

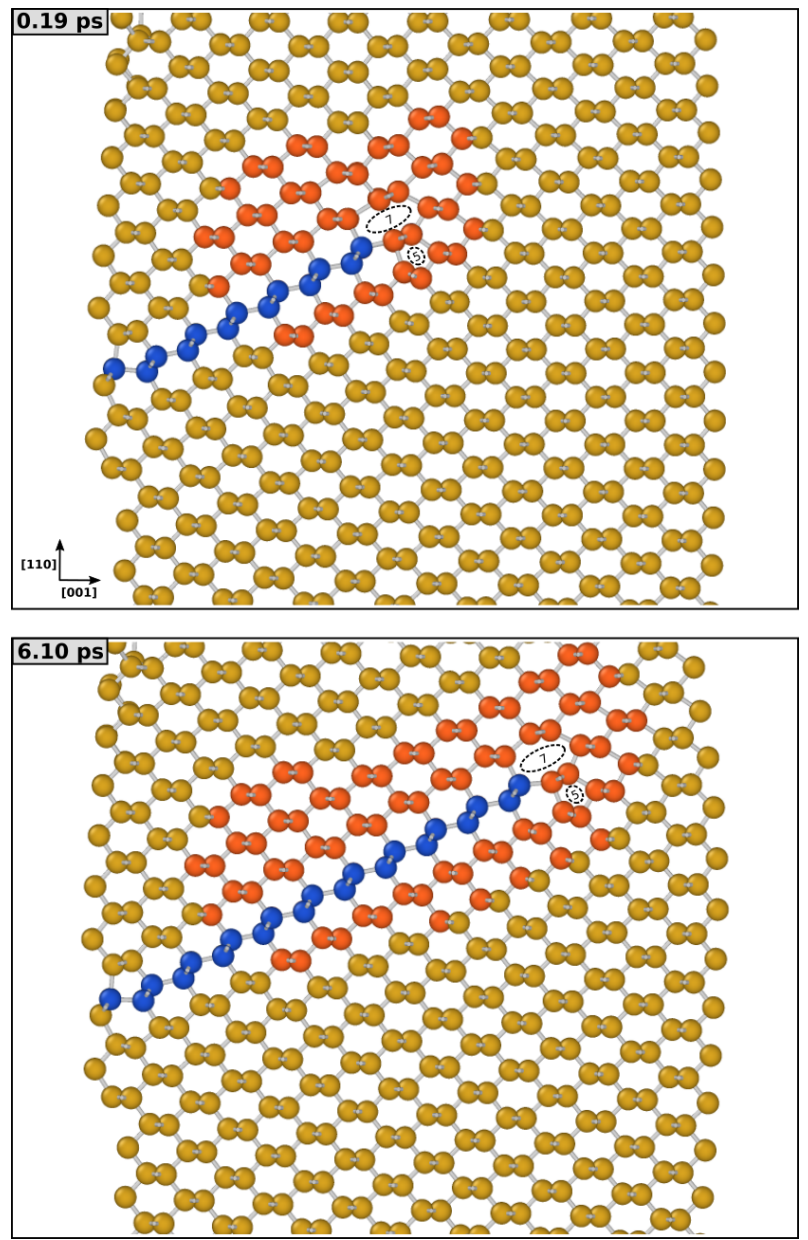

FIG. 3. Dislocation displacement and stacking fault expansion (blue spheres) in a 2D silicon slab, obtained from a LOTF calculation at a $12 \%$ compression strain. Top and bottom images correspond to simulation times of $0.19 \mathrm{ps}$ and $6.10 \mathrm{ps}$, respectively. The atoms represented by red spheres are included in the electronic structure calculation of LOTF.

identical to previously reported core structures [27, 28]. A comparable topology is reported for the edge dislocation 5/7-atom core in $\mathrm{GaN}$ [49] and the $60^{\circ}$ dislocation $\mathrm{G}$ core in silicon [8]. By analogy, one could consider that this dislocation core belongs to the glide set of $\{111\}$ planes. However, it can also be viewed as the sum of two Shockley partials [16, 20, 21] with Burgers vector $1 / 6\langle 112\rangle$, located in the two shuffle planes on both sides of the stacking fault.

The influence of strain rate and temperature was checked with additional calculations. The same deformation mechanism is obtained in all cases, which confirms its dominant role for compression along the $\langle 110\rangle$ orientation. Only the onset of plasticity depends on strain rate and temperature. For a rate equal to $10^{8} \mathrm{~s}^{-1}$ and at $300 \mathrm{~K}$, the first dislocation is nucleated at a strain of $10.43 \%$. Keeping this strain rate but increasing the temperature to $500 \mathrm{~K}$, the first nucleation occurred at a lower strain of $9.52 \%$. This behavior is in agreement with the thermally activated character of dislocation nucleation $[50,51]$. It also suggests that the energy barrier for dislocation nucleation is higher than the one for dislocation migration. In the present conditions, we then obtain an athermal expansion of the dislocation.

\section{B. 2D slab}

We also carry out MD calculations of 2D slabs, which are periodic along $\hat{y}=[1 \overline{1} 0]$ with a single period and compressed along $\hat{z}=[110]$. Nucleated dislocations are then necessarily straight, which allows for an easier analysis of dislocation core motion than in the nanowire. The slab is large enough to ensure that the internal stress state is similar in both the nanowire and the slab during compression. At $600 \mathrm{~K}$, the deformation remains elastic up to a strain equal to $6.9 \%$. Then a dislocation nucleates and propagates in $\{111\}$ planes while leaving an intrinsic stacking fault in its trailing path. This dislocation is the same than those observed during the plastic deformation of nanowires, i.e. it is an edge partial dislocation with a Burgers vector of $1 / 3\langle 112\rangle$. The nucleation strain can be compared to values found in previous investigations. Godet et al. determined a critical strain of $7.0 \%$ at $0 \mathrm{~K}$ for the original SW potential [27], with a similar surface geometry. More recently, Zhang and Cai reported that the same defect could nucleate at $5.6 \%$ at $0 \mathrm{~K}$ for the same potential but with a bent surface step. This value could even be as low as $5.3 \%$ if another potential is used [28]. These results tend to suggest that both the temperature and the interatomic potential have a weaker influence on the nucleation strain than the surface conformation. In fact the nucleation strain is larger for a flat surface, with values greater than $10 \%$. This is not surprising since the critical influence of surface geometry on dislocation nucleation has been largely documented, in particular in $\mathrm{Si}[52]$.

At present the occurrence of stacking fault expansion at room temperature associated to this unusual dislocation has only been observed in classical MD simulations. To corroborate this result, we next carry out LOTF calculations using the $2 \mathrm{D}$ slab model. Our initial configuration is extracted from MD simulations. Dimensions are rescaled to account for lattice parameter differences between MD and LOTF, and residual forces are relaxed at $0 \%$ compression strain. First, we focus on the mechanism leading to dislocation core displacement and stacking fault expansion. The system is compressed at $12 \%$ by reducing the dimension along the $\hat{z}$ axis, and let free to evolve using a damped dynamics algorithm. We observe the displacement of the dislocation core in a $\{111\}$ plane and along a $\langle 112\rangle$ direction, leading to the stacking fault expansion. It takes about $6 \mathrm{ps}$ for the dislocation to travel over $13.32 \AA$ (Fig. 3). The motion is not continuous, with periods where the dislocation seems at rest alternating with displacements of one lattice repetition. 


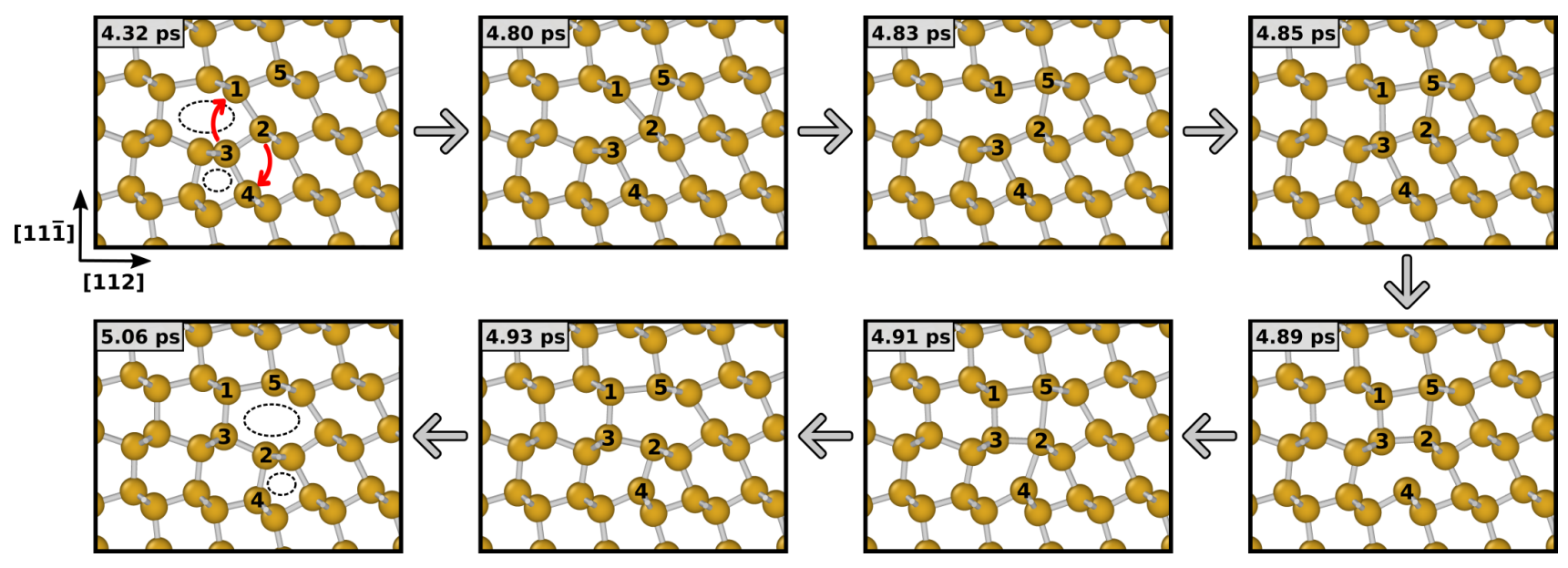

FIG. 4. Sequence of dislocation core geometries extracted from a LOTF calculation (12\% compression, damped dynamics), at various times. Atoms marked 1-5 are those mainly involved in the core displacement (see text for details). A bond is drawn between two atoms when their separation is lower or equal to $2.8 \AA$. Note that the $2 \mathrm{D}$ slab has been rotated along $\hat{y}=[1 \overline{1} 0]$ compared to Fig. 3.

Upon displacements, temperature rises of $30 \mathrm{~K}$ are observed, suggesting a decrease in potential energy. This result confirms that the plasticity mechanism obtained in classical MD calculations is plausible, albeit a large applied strain is required.

The sequence of core atoms displacements leading to dislocation core migration and stacking fault expansion is represented in Fig. 4. At 4.32 ps, the dislocation core is located in a Peierls valley and is close to its stable geometry. LOTF simulations show that the shift of the core to the next valley along $\langle 112\rangle$ requires the rotation along $\hat{y}=[1 \overline{1} 0]$ of the dimer composed of atoms 2 and 3 , which bonds atoms 3 with 1 , and 2 with 4 . The dimer rotation directly leads to the stacking fault expansion. The red arrows in Fig. 4 shows the most straightforward path. However, our calculations reveal that the mechanism is more complex and is decomposed into several elementary steps. The first one, at $4.80 \mathrm{ps}$, is somewhat counter-intuitive. Atom 2 moves close enough to make a bond with atom 5 . Next, the bond between atoms 2 and 1 is loosened (4.83 ps). It allows for atom 1 to connect to atom 3 (4.85 ps). As a consequence, the bond between atoms 3 and 4 breaks ( $4.89 \mathrm{ps}$ ), followed by the creation of a new bond between atoms 2 and 4 (4.91 ps). The last step involves the breaking of the bond between atoms 2 and 5 (4.93 ps). At about $5.06 \mathrm{ps,} \mathrm{the} \mathrm{process} \mathrm{is}$ completed. It is interesting to note that overcoordination is always favored compared to undercoordination during the different steps.

\section{DFT-NEB}

Atomistic simulations of dynamical phenomena, as reported above, are severely limited in timescale. The investigations of dislocation nucleation and propagation

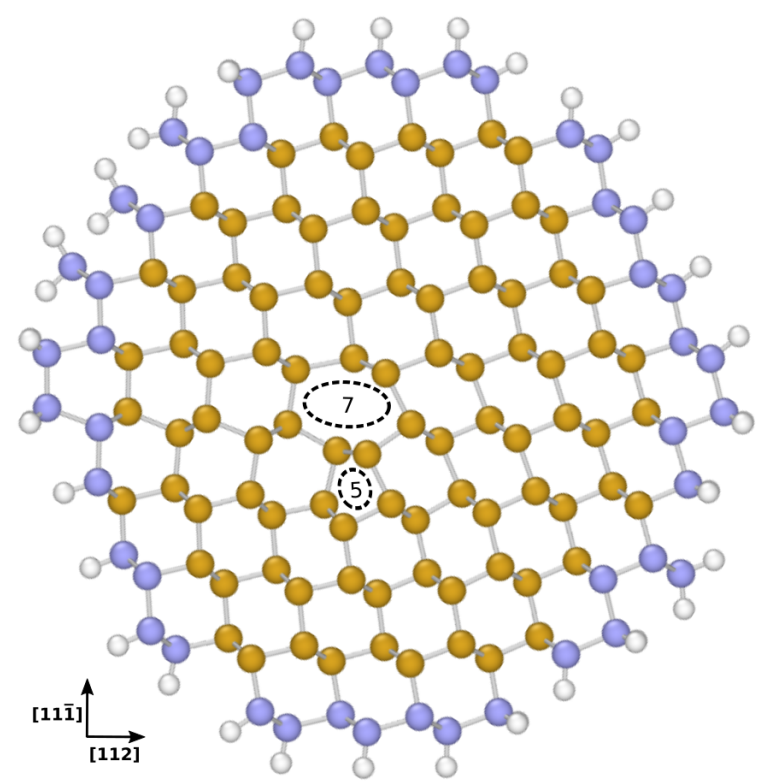

FIG. 5. Initial image for NEB calculation at $10 \%$ compression, obtained by DFT-relaxation. Gold/violet (white) spheres represent silicon (hydrogen) atoms, respectively. Violet atoms were not allowed to move during relaxation.

are then usually restricted to high stress regimes for which the activation energies are low. Such a limitation can be overcome using saddle-point search techniques like NEB in combination with DFT, the results of which are described in this section.

The inputs for DFT-NEB calculations are constructed from the LOTF results at a $12 \%$ compression strain described above (Fig. 4). First, we select two configura- 
tions in which the dislocation is approximately located in two adjacent Peierls valleys, to define the initial and final images in NEB calculations. A homogeneous scaling of the supercell dimension along $\hat{z}$ is made to obtain compression strains lower than $12 \%$. Initial and final configurations are next relaxed at $0 \mathrm{~K}$ with LOTF using a damped dynamics algorithm. Finally, they both include a dislocation core in a Peierls valley, relaxed at the DFT level of accuracy, and embedded in the strain field described by an interatomic potential. In a second step, approximately spherical clusters of 137 silicon atoms are extracted from these two configurations. The clusters center is chosen midway between the dislocation cores in the two geometries. This allows for minimizing boundary effects in the determination of dislocation migration energies [53]. After an appropriate rescaling to take into account the lattice parameters difference between LOTF and DFT, each cluster is next embedded in the orthorhombic supercell described in the section II C. The structure is then periodic along the $\hat{y}$ while a surface is present along the two other directions. Undercoordinated silicon surface atoms are next passivated by hydrogen atoms, initially located at $1.49 \AA$, amounting to 177 atoms in total. The energy of the two configurations is then minimized by atomic relaxation computed by DFT, to obtain the initial and final images for DFTNEB calculations. In these calculations, the positions of silicon atoms close to the surface are not updated, thus ensuring that the strain field exerted on dislocation cores is the same as in LOTF calculations. Fig. 5 shows an example of relaxed configuration.

For a compression strain of $12 \%$, we find that the dislocation core moves to the next Peierls valley for both initial and final images, in agreement with LOTF calculations. This result suggests that such a strain induces a resolved shear stress greater than the Peierls stress of the dislocation. Motionless cores are recovered for compression strains lower or equal to $11 \%$. A geometrical characterization of the dislocation core relaxed with no compression strain is included in the appendix B.

For DFT-NEB calculations, the inputs for the five intermediate images are generated by linear interpolation of the position of all atoms between the initial and final configurations. Both silicon atoms close to the surface and hydrogen atoms are fixed to their initial positions during the NEB relaxation. However, note that the positions of these atoms slightly change through the successive images, which reflects the strain field evolution associated with dislocation migration.

The top graph in Fig. 6 shows the results of the NEB calculations. The energy curves along the minimum energy path (MEP) are characterized by an asymmetric shape, as expected for dislocation displacement under strain $[53,55]$. Also, the maximum energy along the MEP decreases as a function of compression strain. For strains greater than $3 \%$ the minimum energy along the path does not correspond to the final NEB image. We believe that this behavior might be the consequence of the

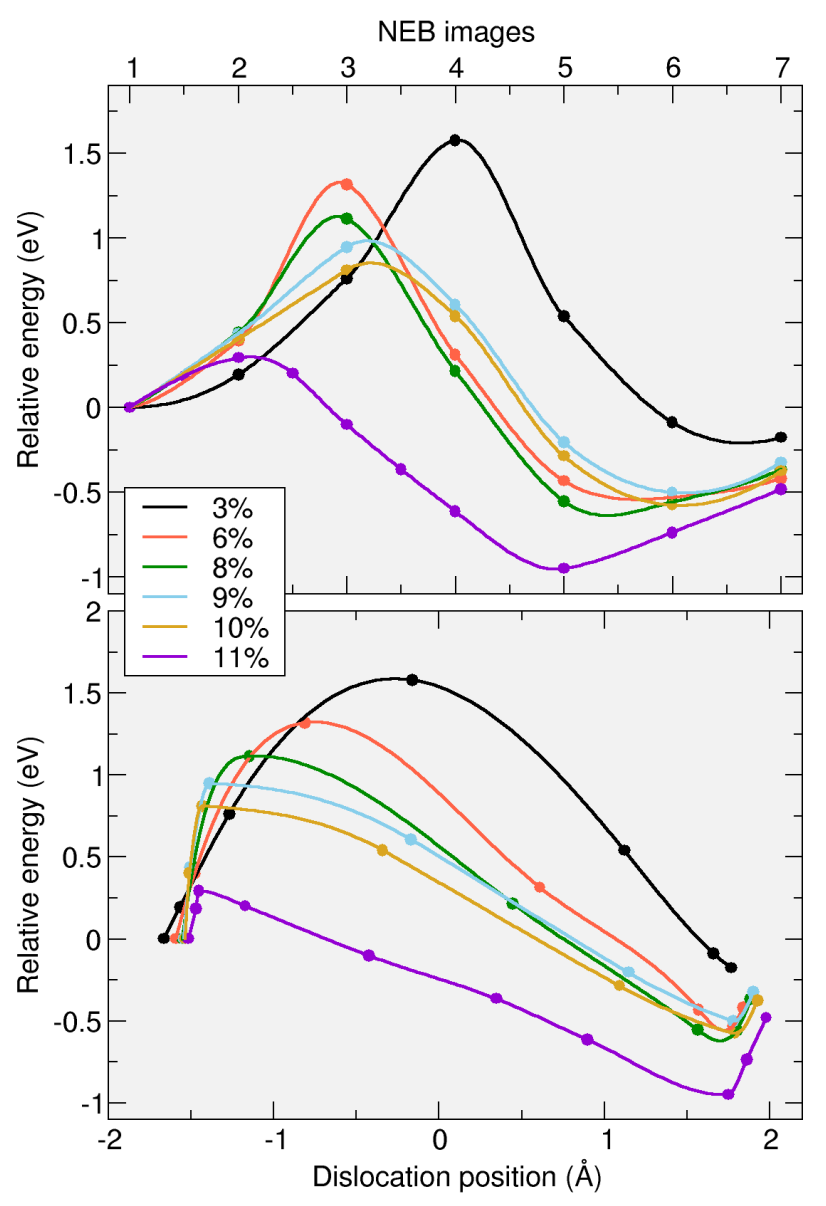

FIG. 6. Variations of relative energy as a function of (top) NEB images (bottom) dislocation core position. Each color corresponds to a different compression strain. Lines connecting dots are guides for the eye obtained from spline-based interpolations. The dislocation position is estimated using the method described in the Appendix C.

use of fixed surfaces with interpolated positions. However, it is assumed to have a negligible influence on the energy barrier. The bottom graph shows the same energy variations but now as a function of the dislocation core position. The latter is determined by monitoring the progressive formation of the stacking fault (Appendix C). The curves suggest that increasing the compression strain displaces the saddle point towards the starting geometry.

The activation energies $\mathrm{Q}(\varepsilon)$ determined as maximums of energy barriers are reported in Fig. 7. As expected, the values decrease for an increasing compression strain and become lower than $1 \mathrm{eV}$ above $9 \%$. These data are fitted using the expression

$$
Q(\varepsilon)=W_{p}\left(1-\frac{\varepsilon}{\varepsilon_{\text {ath }}}\right)^{n}
$$

yielding a Peierls energy $W_{p}=1.744 \mathrm{eV}$, an athermal compression limit $\varepsilon_{\text {ath }}=11.07 \%$, and an exponent 


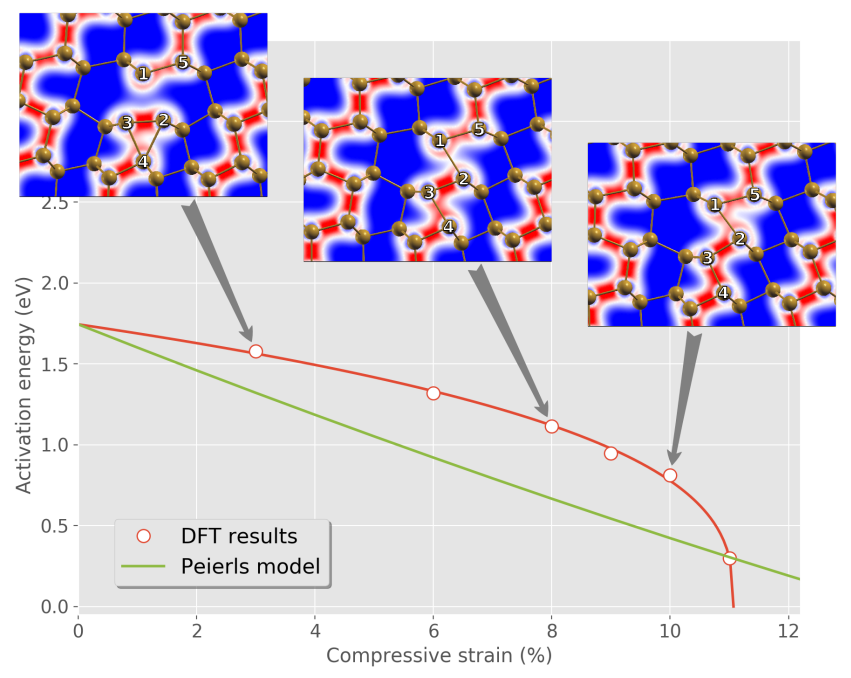

FIG. 7. Activation energy for dislocation displacement as a function of the applied compression strain along $\langle 110\rangle$ (white dots). The red line connecting dots is the result of a fit using Eq. 1. The green line is the activation energy predicted by a Peierls model. The three inset images represent the atomic structure at the saddle point at different compression strains (note that the structures are oriented like in Fig. 4, with the dislocation moving from left to right). A bond is drawn between two atoms when their separation is lower or equal to $2.8 \AA$. Also shown is the electronic density in the (110) plane passing through atoms 1-5 (same atoms as in Fig. 4). The colormap ranges from $0.0 e^{-}$bohr $^{-3}$ (blue) to $0.082 e^{-}$bohr $^{-3}$ (red). Maxima are found in the middle of a bond, with values in excellent agreement with the literature [54].

$n=0.346$. The activation energy as a function of the resolved shear stress $\sigma$ is readily obtained by assuming that $\sigma(\varepsilon)=m E \varepsilon$ (with $m=0.471$ the Schmid factor and $E=170 \mathrm{GPa}$ the elastic modulus for compression along $\langle 110\rangle$ in silicon). Next, the activation volume $\Omega(\sigma)=-\partial Q / \partial \sigma$ can be computed. With no compression, $\Omega$ is equal to $0.122 b^{3}\left(b=a_{0} \sqrt{6} / 3\right)$, which corresponds to a localized atomistic mechanism [56]. Furthermore, we observe that $\Omega(\sigma)$ diverges if $\sigma$ is close to the athermal limit, since $n$ is lower than 1 . It is unclear how this behavior could be explained.

To gain further insights, we compare the DFT results with an ideal Peierls model. For the latter, the energy variation can be written:

$$
E(x, \varepsilon)=\frac{W_{p}}{2}\left(1-\cos \left(\frac{2 \pi x}{d}\right)\right)-\sigma(\varepsilon) b x l
$$

The first term is the Peierls energy approximated by a sinusoid [57]. We use $W_{p}=1.744 \mathrm{eV}$ from the DFT-NEB fit. $x$ is the dislocation position and $d=a_{0} \sqrt{6} / 4$ is the lattice periodicity along $\langle 112\rangle$. The second term is the mechanical work due to dislocation displacement: $b=$ $a_{0} \sqrt{6} / 3$ is the Burgers vector norm and $l=a_{0} / \sqrt{2}$ the dislocation length along the $\hat{y}$ axis. Finally, the resolved shear stress $\sigma(\varepsilon)$ is as previously approximated by $m E \varepsilon$. In principle, one should also add the contribution due to stacking fault expansion $+l x \gamma$. It is neglected here due to the low $\gamma$ value $\left(65 \mathrm{~mJ} \mathrm{~m}^{-2}\right)$ of the silicon intrinsic stacking fault energy [58]. The activation energy for each $\varepsilon$ value is obtained as the maximum of $E(x, \varepsilon)$ for $x \in$ $[0, d]$. The comparison in Fig. 7 shows that the shape of DFT calculated energies is significantly different from the Peierls model, with higher values except for compression strains greater than $11 \%$. It suggests that there is an intricate relation between the lattice friction associated to dislocation displacement and the applied compression.

Figure 7 also shows the geometry and electronic structure at saddle point for three strain values. The electronic structure allows for a finer analysis of the presence of atomic bonds, compared to a simple distance criterion. At $3 \%$ compression strain, the saddle configuration exhibits a clear dangling bond in the core, and is markedly different from all intermediate geometries determined using LOTF (Fig 4). This configuration is obtained by bonding atoms 2 and 4 . The next step is the atom 3 making a bond with atom 1 . This simple two-step mechanism corresponds to the straightforward path depicted by red arrows in Fig. 4. Increasing the compression strain reveals a gradual change of the mechanism. At $6 \%$, it resembles the one at $3 \%$, except that the existence of a dangling bond in the saddle configuration is more ambiguous. At $8 \%$, the saddle configuration in Fig. 7 is topologically equivalent to the initial NEB image, with no broken or new bonds. Finally, at $9 \%-11 \%$, atoms 2 and 5 are much closer than for lower strain values. There is also an increase of electronic density between these atoms, supporting the existence of a bond. For such strain values, the saddle geometry is similar to the LOTF configuration represented in Fig. 4 (4.80 ps). The analysis of the next NEB images confirms that the DFTNEB mechanism at $9 \%-11 \%$ is like the one obtained with LOTF at $12 \%$.

Our DFT-NEB calculations indicate that the mechanism leading to dislocation displacement and stacking fault expansion depends on the applied compression strain. For values lower than $9 \%$, the core dislocation moves by the rotation of the dimer formed by atoms 2 and 3 (red arrows in Fig. 4). This process requires the temporary existence of a dangling bond in the core, an energetically costly defect in silicon, which probably explains the high activation energies. At higher strain values the mechanism depicted in Fig. 4 operates. This mechanism is more complex but without dangling bonds clearly occurring with dislocation displacement. It is noteworthy that atoms 2 and 5 in Fig. 4 are aligned along $\hat{z}$ in two successive (110) atomic layers, and their separation is directly proportional to the applied strain. Increasing the compression along the $\hat{z}$ axis then facilitates the activation of the mechanism and reduces the associated energy. This would explain the change of slope at high strain values of the DFT-NEB activation energy curve in Fig. 7. 


\section{DISCUSSION}

All results obtained in our study demonstrate that the activation of the $1 / 3\langle 112\rangle\{111\}$ slip system can occur in silicon compressed along the $\langle 110\rangle$ orientation. Its partial character leads to the formation and expansion of an intrinsic stacking fault. This key finding raises the question of the significance of this deformation mode. DFT-NEB results indicate that the activation energy is relatively high, being greater than $1 \mathrm{eV}$ when the compression strain is below $9 \%$. We determine that it is equal to $1.74 \mathrm{eV}$ at zero strain. Such high values are conflicting with an activation at room temperature, making high stress/strain conditions a necessary requirement. It is obviously not possible in bulk silicon, which fails in a brittle manner due to the formation and propagation of cracks. However, large strain/stresses values have been reported in various nanostructures $[13,59-63]$. In these systems, the occurrence of the slip system studied here cannot be excluded.

Several experiments reported the presence of stacking faults in silicon nanostructures. For instance, Wagner and co-workers observed by transmission electron microscopy the presence of a stacking fault created during the flat punch compression of silicon blunt nanocubes at room temperature [17]. They assumed that it was produced by the propagation of a single glide partial dislocation. It is conceivable that such a dislocation could nucleate in a region where a beta-tin phase transition occurred [64]. While there is no definite proof, it is probably different from the mechanism described here, since the compression orientation was $\langle 100\rangle[17]$. The compression of $\langle 110\rangle$ oriented silicon nanopillars was investigated by Merabet et al. [16, 20]. Their transmission electron microscopy observations revealed the presence of stacking faults, tentatively explained by the dissociation of shuffle $60^{\circ}$ dislocations into shuffle partial dislocations [21]. This latter mechanism might also explain the formation of stacking faults during compression of $\langle 111\rangle$ oriented nanopillars [65]. Both nucleation from the betatin phase and shuffle dislocation dissociation have been recently discussed by J. Rabier [66]. Our work provides an original and alternative explanation for the formation of stacking faults in silicon at small scales.

Another interesting aspect concerns the current terminology at use for dislocations in silicon. Since the pioneering work by Hornstra [9], it is common to distinguish dislocations depending whether they belong to narrowly spaced $\{111\}$ 'glide' planes or widely spaced $\{111\}$ 'shuffle' planes [57]. Until ten years ago, the situation was thought to be relatively simple with dissociated glide and perfect shuffle set dislocations operating at high and low temperatures, respectively [7]. New experimental and theoretical data tend to suggest a greater intricacy, especially at small scale. We already discussed mounting evidences of stacking faults and dissociated dislocations at low temperature $[16,17,20,63-65]$. The distinction between glide and shuffle set dislocations also seems to dilute. It is in fact difficult to decide in which set of planes the dislocation investigated in this work belongs to, because the core spreads over several glide and shuffle planes. Note that the same can be said of the S3 core configuration for the $60^{\circ}$ dislocation [6]. These dislocation cores are spread out enough to be described as shuffleglide complexes, borrowing the terminology introduced by Zhang and Cai [28].

\section{CONCLUSION}

We report the formation of a stacking fault in silicon compressed along $\langle 110\rangle$ at low temperature, thanks to an original plasticity mechanism. A partial edge dislocation of Burgers vector equal to $1 / 3\langle 112\rangle$ nucleates and slips in $\{111\}$ planes, leaving an intrinsic stacking fault behind. These results are obtained by combining three different simulation methods, molecular dynamics, learn on the fly, and density functional theory. We fully characterize the dislocation core geometry and mobility. In particular, we describe the atomistic mechanism allowing for dislocation displacement and stacking fault expansion. We find that this mechanism changes according to the compression strain. At low strain values the dislocation core displacement is associated with the creation of a dangling bond, with a large energy barrier which inhibits its activation. For compression strains greater than about $8 \%$, the reduction of the interlayer distance allows for a more complex displacement mechanism with no dangling bond in the dislocation core and a significant decrease of the activation energy. Our results then offer a potential explanation for the observation of stacking faults in $\langle 110\rangle$ compressed silicon at small scales and low temperature.

\section{Appendix A: LOTF}

The hybrid LOTF technique allows for carrying out molecular dynamics using forces coming from an adjustable classical potential form. The main idea of the technique is to update the adjustable potential from the knowledge of reference quantum forces calculated in a quantum region (red atoms in Fig. 8) and from classical reference forces elsewhere (light cyan and gold atoms in Fig. 8). Practically, the adjustable potential is obtained by tuning locally the parameters associated with pair and triplet interactions in order to reproduce at best the reference forces in the fitting region. The fitting region where the potential is updated contains the quantum region plus a surrounding classical buffer region (light cyan atoms in Fig. 8) including all atoms within a radius of $R_{f i t}=10 \AA$ from the quantum region. A fitting region larger than the quantum region allows for a smooth variation of the potential parameters from the active region to the bulk. The buffer region also helps to reduce and spread the fitting errors. The LOTF technique proceeds by periodic updates of the adjustable potential, with the 
(a)

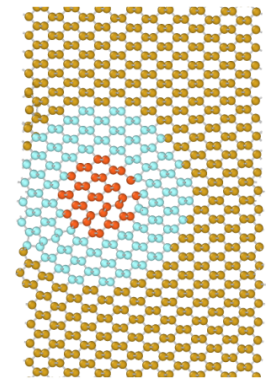

(b)

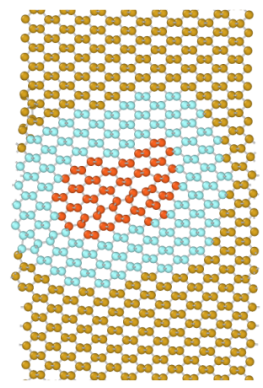

(c)

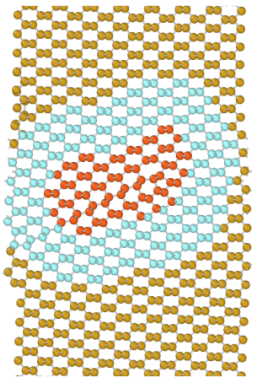

FIG. 8. (a): Structure of the initial configuration of the 1206 atoms slab under $12 \%$ compression. The colors indicate the different regions used in the hybrid LOTF scheme, red: quantum region (reference forces calculated from DFTSIESTA[67]), light cyan: classical part of the fitting region (reference forces calculated from a classical potential), gold: classical region (forces calculated from a classical potential). The fitting region encompasses the red and light cyan atoms. (b) Configuration obtained from (a) after $3.0 \mathrm{ps}$ of LOTF damped dynamics. (c) Configuration obtained from (a) after 4.1 ps of LOTF damped dynamics.

quantum and fitting regions both dynamically evolving to follow the defect propagation. The adjustable potential is updated through a series of extrapolation/interpolation dynamical stretches separated by a periodic evaluation of the reference forces and by a periodic force fitting (see [36, 37, 39] for more details). The quantum and fitting regions are updated using simple topological arguments, detailed below.

The quantum reference forces on each atom of the quantum region are approximated by separated quantum calculations done with the SIESTA [67] approach to density functional theory (with the same technical details as in [39]). For each atom in the quantum region we carve out a cluster of $6.6 \AA$ radius from the main system and terminate the cut bonds with hydrogen atoms. After the SIESTA calculation of the cluster, the force on the central atom is retained in the list of the reference forces. Both the classical potential of reference as well as the adjustable potential have a Stillinger-Weber form [68] (the technical details are given in [39] and in its supplementary material). We recall that out of the fitting region (gold atoms in Fig. 8), the adjustable potential has fixed parameters identical to the classical potential of reference. The adjustment is restricted to the fitting region.

An example of an initial configuration is shown in figure $8(\mathrm{a})$, with a defect already present inside the bulk. The initial quantum region is a cylinder of $7.5 \AA$ centered around an atom in the vicinity of the defect. Starting from this initial set up, the selection algorithm detects all the coordination changes through the analysis of the neighbor lists of the quantum atoms at the boundary of the quantum and buffer region. Whenever a change is detected, all the neighbors of the concerned quantum atoms are incorporated to the quantum region. Following this

algorithm, the quantum and buffer regions grow when the defect propagates. As it can be seen from Fig. 8(b) and $8(\mathrm{c})$ this selection scheme nicely follows the defect propagation and quickly releases the relative arbitrariness of the initial quantum region.

\section{Appendix B: Dislocation core geometry}

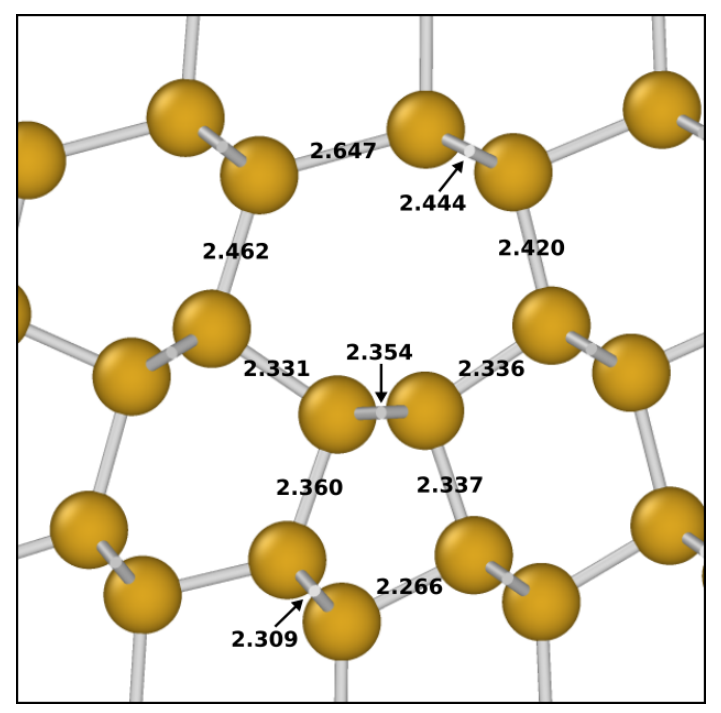

FIG. 9. Zoomed view of the $1 / 3\langle 112\rangle$ partial dislocation core relaxed by DFT calculations. The numbers indicate the bond lengths (in $\AA$ ) between silicon atoms, represented by gold spheres.

In this section, we report the geometry of the $1 / 3\langle 112\rangle$ partial dislocation core. It is obtained by relaxing with DFT an initial configuration built from a LOTF-relaxed system at $0 \%$ compression. The converged core structure is shown in Fig. 9.

The dislocation core structure includes two rings of respectively seven and five $\mathrm{Si}$ atoms. All the atoms belonging to these rings are four-fold coordinated. These characteristics make this dislocation core apparently similar to the $60^{\circ}$ perfect glide dislocation core [8], except that this one does not allow for stacking fault formation and expansion. The bond lengths in the core are also reported in Fig. 9. Except for one, values range from 2.309 to $2.462 \AA$, indicating moderate strains by comparison to the bulk DFT value of $2.368 \AA$. Finally, note that the lack of coordination defects prevents a possible reconstruction along the dislocation line. This assertion was confirmed by additional DFT calculations using initial configurations with a double period along the $\hat{y}$ axis. 


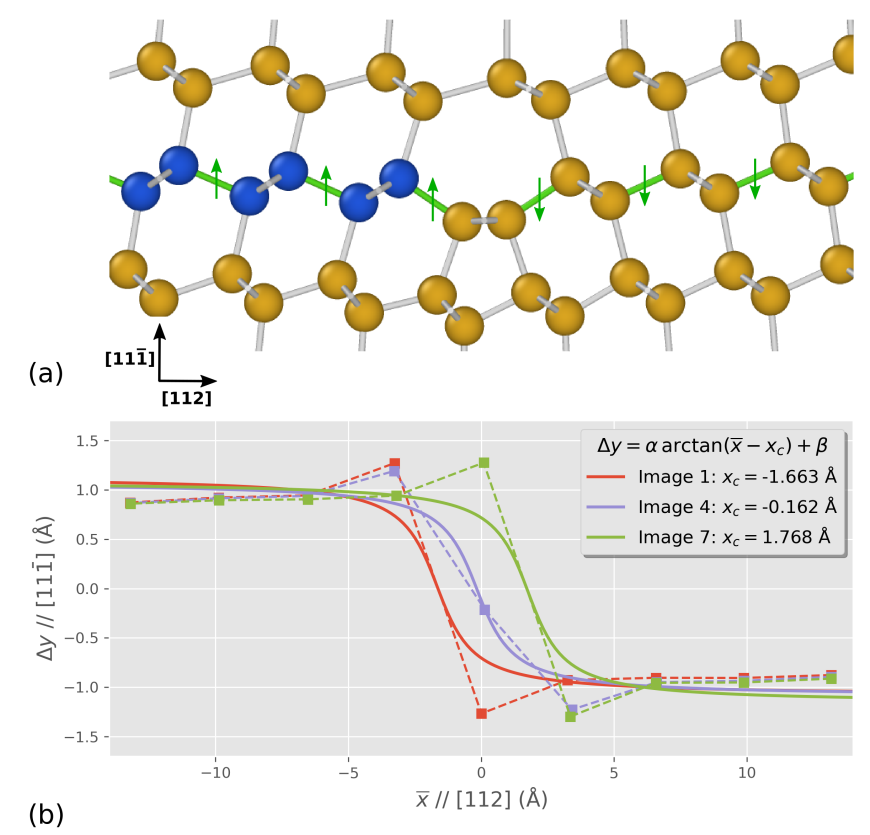

FIG. 10. (a) Dislocation core configuration for a $3 \%$ compression. Blue spheres represent silicon atoms bordering the stacking fault, and green tubes show bonds used for dislocation position calculation. (b) The squares represent atomic $\mathrm{y}$-coordinate differences $\Delta y$ along the $[11 \overline{1}]$ axis as a function of atomic x-coordinate averages along the [112] axis, for atoms bonded by green tubes in (a). The dashed lines are guides for the eye. The colors correspond to three different calculated NEB images, for a $3 \%$ compression. The full lines show the result of the fit using the elasticity expression reported in the legend of the figure.

\section{Appendix C: Determination of dislocation position}

Final images relaxed using NEB-DFT calculations are linearly spaced in configuration space (except the climbing image associated to the saddle state). It obviously does not mean that there is a constant distance between dislocation core centers in successive configurations. This issue is documented in the literature $[53,55]$.
In principle the dislocation core position could be determined by fitting the calculated atomic displacements with the strain field given by elasticity theory, but this approach is difficult to implement for the small systems used in this work. An alternative strategy relies on monitoring the variation of a well chosen geometrical quantity [53]. For instance, a possible indicator for the $1 / 3\langle 112\rangle$ dislocation core is the atomic coordinate difference along the [11]] axis between successive atoms along [112] which form 'long' bonds (colored in green in Fig. 10a). This quantity varies from a positive to a negative value due to the formation of the stacking fault in the wake of the dislocation (green arrows in Fig. 10-a), and can then be used to define a dislocation core center.

Figure 10-b shows computed values for the initial, final, and saddle-state images for a $3 \%$ compression strain. These variations are fitted using the expression $\alpha \arctan \left(\bar{x}-x_{c}\right)+\beta$ derived from elasticity theory [57]. Here $\bar{x}$ is the center of the bond along the $\mathrm{x}$-axis, and $\alpha, \beta$, and $x_{c}$ are fitting parameters. One can see in Fig. 10 that the above expression is maybe too simple for a perfect fit. Nevertheless, assuming that the dislocation center is at $x_{c}$, reasonable dislocation core positions are obtained. For instance, the distance traveled by the dislocation core from the initial to the final NEB image is calculated to be equal to $3.43 \AA$, which is slightly larger than the period along $[112]\left(\sqrt{6} a_{0} / 4=3.35 \AA\right)$. The difference is further reduced if one takes into account the Poisson expansion along [112] due to the compression.

\section{ACKNOWLEDGMENTS}

The classical MD and DFT-NEB calculations were performed at the Mesocentre SPIN at the University of Poitiers. We gratefully acknowledge financial support by the French national research agency (ANR) within the project "BrIttle to DUctile transition in silicon at Low dimensions" under the grant reference ANR-12-BS04-000301. Part of this work was done in the kind memory of Alessandro De Vita, inventor the LOTF technique in 1996. Forever remembered, forever missed.
[1] I. Ray and D. Cockayne, Proc. R. Soc. Lond. A 325, 543 (1971).

[2] B. Joós, Q. Ren, and M. S. Duesbery, Phys. Rev. B 50, 5890 (1994).

[3] V. V. Bulatov, J. F. Justo, W. Cai, S. Yip, A. S. Argon, T. Lenosky, M. de Koning, and T. D. de la Rubia, Philos. Mag. A 81, 1257 (2001).

[4] J. Rabier, P. Cordier, J. L. Demenet, and H. Garem, Mater. Sci. Eng. A 309-310, 74 (2001).

[5] L. Pizzagalli, P. Beauchamp, and J. Rabier, Philos. Mag. A 83, 1191 (2003).

[6] L. Pizzagalli, J. Godet, and S. Brochard, Phys. Rev. Lett.
103, 065505 (2009).

[7] J. Rabier, L. Pizzagalli, and J.-L. Demenet, in Dislocation in solids, Vol. 16, edited by L. Kubin and J. P. Hirth (Elsevier, 2010) Chap. 93, p. 47.

[8] D. Rodney, L. Ventelon, E. Clouet, L. Pizzagalli, and F. Willaime, Acta Mater. 124, 633 (2017).

[9] J. Hornstra, J. Phys. Chem. Solids 5, 129 (1958).

[10] M. S. Duesbery and B. Joós, Philos. Mag. Lett. 74, 253 (1996).

[11] L. Pizzagalli and P. Beauchamp, Philos. Mag. Lett. 88, 421 (2008).

[12] H. E, C. Teresi, and W. Gerberich, Metall. Mater. Trans. 
A 47, 5839 (2016).

[13] F. Östlund, K. Rzepiejewska-Malyska, K. Leifer, L. M. Hale, Y. Tang, R. Ballarini, W. W. Gerberich, and J. Michler, Adv. Funct. Materials 19, 2439 (2009).

[14] W. W. Gerberich, D. D. Stauffer, A. R. Beaber, and N. I. Tymiak, J. Mater. Research 27, 552 (2012).

[15] F. Abed El Nabi, J. Godet, S. Brochard, and L. Pizzagalli, Modelling Simul. Mater. Sci. Eng. 23, 025010 (2015).

[16] A. Merabet, M. Texier, C. Tromas, S. Brochard, L. Pizzagalli, L. Thilly, J. Rabier, A. Talneau, Y.-M. L. Vaillant, O. Thomas, and J. Godet, Acta Mater. 161, 54 (2018).

[17] A. J. Wagner, E. D. Hintsala, P. Kumar, W. W. Gerberich, and K. A. Mkhoyan, Acta Mater. 100, 256 (2015).

[18] D. Kilymis, C. Gérard, J. Amodeo, U. Waghmare, and L. Pizzagalli, Acta Mater. 158, 155 (2018).

[19] M. Gordon, T. Baron, F. Dhalluin, P. Gentile, and P. Ferret, Nanoletters 9, 525 (2009).

[20] M. Texier, A. Merabet, C. Tromas, S. Brochard, L. Pizzagalli, L. Thilly, J. Rabier, A. Talneau, Y.-M. L. Vaillant, O. Thomas, and J. Godet, J. Phys. Conf. Series 1190, 012004 (2019).

[21] F. Louchet and J. Thibault-Desseaux, Revue de Physique Appliquée 22, 207 (1987).

[22] H. Zhang, J. Tersoff, S. Xu, H. Chen, Q. Zhang, K. Zhang, Y. Yang, C.-S. Lee, K.-N. Tu, J. Li, and Y. Lu, Science Advances 2, e1501382 (2016).

[23] C. K. Chan, H. Peng, G. Liu, K. McIlwrath, X. F. Zhang, R. A. Huggins, and Y. Cui, Nature Nanotechnology 3, 31 (2008).

[24] X. H. Liu, F. Fan, H. Yang, S. Zhang, J. Y. Huang, and T. Zhu, ACS Nano 7, 1495 (2013), pMID: 23272994.

[25] A. Pedersen, M. Bieri, M. Luisier, and L. Pizzagalli, Phys. Rev. Applied 7, 054012 (2017).

[26] J. Li, Z. Shan, and E. Ma, Mater. Res. Soc. Bull. 39, 108 (2014).

[27] J. Godet, L. Pizzagalli, S. Brochard, and P. Beauchamp, Comp. Mat. Sci. 30, 16 (2004).

[28] X. Zhang and W. Cai, (2018), arXiv:1806.01974 [physics.app-ph].

[29] S. Plimpton, J. Comput. Phys. 117, 1 (1995).

[30] Http://lammps.sandia.gov.

[31] L. Pizzagalli, J. Godet, J. Guénolé, S. Brochard, E. Holmstrom, K. Nordlund, and T. Albaret, Journal of Physics: Condensed Matter 25, 055801 (2013).

[32] Each atom moving beyond the flat punch position $z_{0}$ feels a repulsive force perpendicular to the flat punch and with a magnitude given by $F(z)=K\left(z-z_{0}\right)^{2}$. It corresponds to the command FIX INDENT in the LAMMPS code [30].

[33] S. Nosé, in Computer Simulation in Materials Science, NATO ASI Series E: Applied Sciences, Vol. 205, edited by M. Meyer and V. Pontikis (Kluwer Academic, Dordrecht, 1991) p. 21.

[34] W. G. Hoover, Phys. Rev. A 31, 1695 (1985).

[35] J. Godet, S. Brochard, L. Pizzagalli, P. Beauchamp, and J. M. Soler, Phys. Rev. B 73, 092105 (2006).

[36] G. Csányi, T. Albaret, M. C. Payne, and A. De Vita, Phys. Rev. Lett. 93, 175503 (2004).

[37] G. Csányi, T. Albaret, G. Moras, M. C. Payne, and A. D. Vita, Journal of Physics: Condensed Matter 17, R691 (2005).

[38] J. Kermode, T. Albaret, D. Sherman, N. Bernstein,
P. Gumbsch, M. C. Payne, G. Csányi, and A. De Vita, Nature 455, 1224 (2008).

[39] D. Fernandez-Torre, T. Albaret, and A. De Vita, Phys. Rev. Lett. 105, 185502 (2010).

[40] P. Giannozzi, O. Andreussi, T. Brumme, O. Bunau, M. B. Nardelli, M. Calandra, R. Car, C. Cavazzoni, D. Ceresoli, M. Cococcioni, N. Colonna, I. Carnimeo, A. D. Corso, S. de Gironcoli, P. Delugas, R. A. DiStasio, A. Ferretti, A. Floris, G. Fratesi, G. Fugallo, R. Gebauer, U. Gerstmann, F. Giustino, T. Gorni, J. Jia, M. Kawamura, H.-Y. Ko, A. Kokalj, E. Küçükbenli, M. Lazzeri, M. Marsili, N. Marzari, F. Mauri, N. L. Nguyen, H.V. Nguyen, A. O. de-la Roza, L. Paulatto, S. Poncé, D. Rocca, R. Sabatini, B. Santra, M. Schlipf, A. P. Seitsonen, A. Smogunov, I. Timrov, T. Thonhauser, P. Umari, N. Vast, X. Wu, and S. Baroni, Journal of Physics: Condensed Matter 29, 465901 (2017).

[41] J. P. Perdew, K. Burke, and M. Ernzerhof, Phys. Rev. Lett. 77, 3865 (1996).

[42] P. E. Blöchl, Phys. Rev. B 50, 17953 (1994).

[43] H. J. Monkhorst and J. D. Pack, Phys. Rev. B 13, 5188 (1976).

[44] M. Methfessel and A. T. Paxton, Phys. Rev. B 40, 3616 (1989).

[45] H. Jónsson, G. Mills, and K. W. Jacobsen, in Classical and Quantum Dynamics in Condensed Phase Simulations, edited by B. J. Berne, G. Ciccotti, and D. F. Coker (World Scientific, 1998) Chap. 16, p. 385.

[46] G. Henkelman, B. P. Uberuaga, and H. Jónsson, J. Chem. Phys. 113, 9901 (2000).

[47] P. Mahler Larsen, S. Schmidt, and J. Schiøtz, Modelling Simul. Mater. Sci. Eng. 24, 055007 (2016).

[48] S. Korte, J. Barnard, R. Stearn, and W. Clegg, Int. J. of Plasticity 27, 1853 (2011).

[49] I. Belabbas, P. Ruterana, J. Chen, and G. Nouet, Philos. Mag. 86, 2241 (2006).

[50] T. Zhu, J. Li, A. Samanta, A. Leach, and K. Gall, Phys. Rev. Lett. 100, 025502 (2008).

[51] S. Brochard, P. Hirel, J. Godet, and L. Pizzagalli, Acta Mater. 58, 4182 (2010).

[52] J. Godet, L. Pizzagalli, S. Brochard, and P. Beauchamp, Phys. Rev. B 70, 054109 (2004).

[53] L. Pizzagalli, P. Beauchamp, and H. Jónsson, Philos. Mag. 88, 91 (2008).

[54] Z. W. Lu, A. Zunger, and M. Deutsch, Phys. Rev. B 47, 9385 (1993).

[55] D. Rodney and L. Proville, Phys. Rev. B 79, 094108 (2009).

[56] J. Li, Mater. Res. Soc. Bull. 32, 151 (2007).

[57] J. P. Hirth and J. Lothe, Theory of dislocations, edited by 2nd edition (Wiley, New York, 1982).

[58] L. Kubin, Dislocations, mesoscale simulations and plastic flow, edited by A.P.Sutton and R. Rudd, Oxford Series on Materials Modelling (Oxford University Press, London, 2013) p. 238.

[59] T. Kizuka, Y. Takatani, K. Asaka, and R. Yoshizaki, Phys. Rev. B 72, 035333 (2005).

[60] X. Han, K. Zheng, Y. Zhang, X. Zhang, Z. Zhang, and Z. L. Wang, Adv. Mater. 19, 2112 (2007).

[61] Y. Zhu, F. Xu, Q. Qin, W. Y. Fung, and W. Lu, Nanoletters 9, 3934 (2009).

[62] J. Rabier, A. Montagne, J. L. Demenet, J. Michler, and R. Ghisleni, Phys. Stat. Sol. (c) 10, 11 (2013).

[63] Y. Hong, N. Zhang, and M. A. Zaeem, Acta Mater. 145, 
8 (2018).

[64] K. Mylvaganam and L. Zhang, Scripta Materialia 65, 214 (2011).

[65] Y. He, L. Zhong, F. Fan, C. Wang, T. Zhu, and S. X. Mao, Nature Nanotechnology 11, 866 (2016).

[66] J. Rabier, Materials Today Proceedings 5, 14705 (2018).
[67] J. M. Soler, E. Artacho, J. D. Gale, A. García, J. Junquera, P. Ordejón, and D. Sánchez-Portal, J. Phys.: Condens. Matter 14, 2745 (2002).

[68] F. H. Stillinger and T. A. Weber, Phys. Rev. B 31, 5262 (1985). 